\title{
Specific rearrangement of the microtubular network during the cell cycle phases and correlation with micronucleation and death induced by anticancer drugs in NIH/3T3 subcultures
}

\author{
ALESSANDRA SPANO $^{1}$, GIANNI MONACO ${ }^{1}$, SERGIO BARNI $^{2}$ and LUIGI SCIOLA ${ }^{1}$ \\ ${ }^{1}$ Department of Physiological, Biochemical and Cellular Sciences, University of Sassari, \\ Via Muroni 25, Sassari I-07100; ${ }^{2}$ Department of Animal Biology and CNR Center for \\ Histochemistry, University of Pavia, Piazza Botta 10, Pavia I-27100, Italy
}

Received April 2, 2008; Accepted July 19, 2008

DOI: $10.3892 /$ or_00000158

\begin{abstract}
The changes of the microtubular network induced by microtubule destabilizing (Vinblastine, VBL) and stabilizing (Taxol, TAX) agents were studied in NIH/3T3 fibroblastic cells in conventional culture conditions $(\mathrm{NIHb})$ and in a subpopulation (NIHs), obtained after serum deprivation and expressing different morphofunctional features and higher cytokinetic activity. In this cell model, we analyzed VBL and TAX effects on cell cycle and microtubular network, in relation to cell death. In NIHb cells, VBL induced higher microtubule depolymerization, prevalence of tubulin paracrystals and micronucleation, while, in NIHs cells, lower depolymerization and appearance of tubulin spiral-like structures, with lower micronucleation, increase of apoptosis and disappearance of high polyploid cells. DNA static cytofluorometry of cells showing paracrystals or spirals permitted correlation of the appearance of these tubulin aggregation forms with the cell cycle phases. In NIHb cultures, the DNA content curves, in cells with paracrystals or spirals, showed a similar trend, with a higher frequency of the two anomalies in the G2/M phase. In NIHs cultures, paracrystals and spirals are found in G2/M cells, while G1 cells showed prevailingly paracrystals. TAX induced the appearance of microtubule bundles in the two cultures. The prevalence of circular bundles was found in NIHb cells, while a higher number of linear bundles was shown in NIHs cells. In NIHb cells, circular bundles were related to higher
\end{abstract}

Correspondence to: Dr Luigi Sciola, Department of Physiological, Biochemical and Cellular Sciences, University of Sassari, Via Muroni 25, Sassari I-07100, Italy

E-mail: sciola@uniss.it

Key words: NIH/3T3 subcultures, antimicrotubule drugs, paracrystals, spiral-like structures, apoptosis, micronucleation apoptosis and micronucleation. DNA cytofluorometry, in cells with linear or circular bundles, showed that the latter was present with high frequency in NIHb cells in all the cell cycle phases; in NIHs cells, they appeared, with lower frequency, prevailing in the S-G2/M phase. Furthermore, in NIHs cells, the appearance of linear bundles in G1 cells was related to a lower micronucleation. These finding showed that microtubule reorganization in different cell cycle phases could play a role in the progression of nuclear fragmentation/ micronucleation relating to cell death.

\section{Introduction}

Microtubules are important cytoskeletal components involved in many cellular events (e.g. mitosis, organelle dislocation and cell locomotion). The necessity of microtubules for cell survival, coupled with the need to maintain a delicate balance between free and assembled tubulin, makes these structures an interesting target for many drugs used for cancer chemotherapy. Antimicrotubule agents including depolymerizing drugs (vinblastine, vincristine and vinorelbine) and polymerizing agents (paclitaxel and docetaxel) are widely used either alone or in combination with other anticancer drugs. Moreover, these antimicrotubule agents are promoters of apoptosis in cancer cells $(1,2)$.

The Vinca alkaloids, such as vinblastine, widely used for the treatment of leukemia, lymphomas, and some solid tumors (1), induce the destabilization of polymerized tubulin by blocking the region involved in tubulin dimer attachment, thus preventing polymerization of microtubules (2). On the contrary, taxanes bind tubulin by stabilizing the microtubule polymer, and promoting the formation of microtubule bundles (3-5).

It has generally been believed that the antitumor effects of these toxins mainly depend on interference with the normal function of microtubules and blockage of cell cycle progression in the G2-M phase. Suppression of spindle dynamics by low concentrations of drugs is a common mechanism responsible for mitotic block, induced by microtubule-stabilizing and -destabilizing agents $(6,7)$. 
Several laboratories demonstrated that, at clinically relevant concentrations, Vinca alkaloids are able to induce apoptotic cell death in several solid tumor cells $(2,8)$. Cell death also occurs in cancer cells treated with Taxol (9).

Nevertheless, the relationship between mitotic block and cell death induced by different antimicro-tubule agents must be defined. As far as Taxol is concerned, although apoptosis may occur after a prior mitotic arrest (10), it could also be induced independently of G2-M arrest (11). Previous studies with lung carcinoma A549 cells found that low concentrations of Taxol inhibited cell proliferation without blocking cells at mitosis (12) and induced a large population of hypodiploid cells seen close to the G1 peak (13). It was speculated that the aneuploid cells may originate from aberrant mitosis (13), although the mechanism remained to be determined.

Furthermore, previous studies have revealed that glucocorticoids could selectively inhibit paclitaxel-induced apoptotic cell death, but did not affect the ability of paclitaxel to induce microtubule bundling and mitotic arrest $(14,15)$.

On the other hand, the combined use of paclitaxel and Vinca alkaloids in clinical trials suggested that the cooperative effects of the drugs on apoptosis are not mediated through similar disruptional effects on microtubules (16). These phenomena suggested that antimicrotubule agent induced apoptosis may take place via a separate pathway independent of cell cycle arrest and microtubule alterations (17). Further variability in the drug responses could derive from the different cytokinetic activity and the different level of microtubule network organization.

Starting from these assumptions, the aim of this study was to correlate the specific changes of the microtubular network induced by depolymerizing (VBL) and polymerizing (TAX) agents with the specific phases of the cell cycle and their sensitivity to cell death events. For this purpose, we used two NIH/3T3 cultures with different proliferative activity, derived from cells in normal culture conditions (NIHb) or obtained after critical culture conditions (NIHs), respectively.

NIHs cells, obtained from the mouse NIH/3T3 cell line after prolonged serum deprivation, represent a particularly resistant surviving subpopulation with a higher proliferative activity. These cells, that maintain the fibroblastic morphology, showed lower dimension in the modal population, even though aneuploid/polyploid cells were present in subconfluent cultures (18).

From our point of view, NIH/3T3 cells, in the two experimental conditions, could represent a cell model in which it is possible to examine and compare some changes in part similar to those occurring during neoplastic transformation (18).

\section{Materials and methods}

Cell cultures. NIH/3T3 cells, a mouse fibroblastic line, were used in normal (NIHb) and after critical culture conditions (NIHs). Starting from the conventional culture (NIHb), we selected, after prolonged (7-10 days) serum deprivation (culture medium containing $0.5 \%$ foetal calf serum) and massive detachment from the growth substratum, a subpopulation of resistant cells (NIHs), characterized by high proliferative activity and different morphology (18), in comparison to those in basal culture condition (NIHb).
In the experiments performed in this work, all cultures were maintained in Dulbecco's modified Eagle's medium supplemented with $10 \%$ foetal calf serum, $2 \mathrm{mM}$ L-glutamine, $100 \mathrm{U} / \mathrm{ml}$ penicillin, and $100 \mu \mathrm{g} / \mathrm{ml}$ streptomycin (Invitrogen Ltd., Paisley, UK). The cells were cultured at $37^{\circ} \mathrm{C}$ in a humidified chamber containing $5 \% \mathrm{CO}_{2}$, and analyzed, as sub-confluent monolayer, in control and after treatment conditions.

Drug treatment. Cell cultures were treated for $24 \mathrm{~h}$ with the antimicrotubular agents Vinblastine sulphate (VBL) and Taxol (TAX) at concentrations of 8 and $10 \mu \mathrm{g} / \mathrm{ml}$ in complete medium, respectively.

Tubulin and DNA labelling. After washing in PBS, NIHb and NIHs cells, grown on glass coverslips, were fixed in 3\% PFA in PBS containing $1 \mathrm{mM}$ EGTA and $1 \mathrm{mM} \mathrm{MgCl}_{2}$ (buffer A) for $30 \mathrm{~min}$ at room temperature. After fixation and washing in buffer A, the cells were permeabilized with Triton X-100 $(0.2 \%$ in buffer $A)$ for 15 min at room temperature. Cells were incubated with anti- $\beta$-tubulin primary antibody (dilution $1: 400$ ) for $45 \mathrm{~min}$ in a humid chamber. After washing in buffer A, the cells were incubated with FITC-conjugated secondary antibody (dilution 1:250) for $45 \mathrm{~min}$ in a humid chamber. In indirect immunofluorescence techniques, negative reaction controls were obtained by omitting the incubation in the presence of the primary antibody. In all fluorescence microscopy specimens, nuclear counter-staining was obtained with Hoechst $33342(2 \mu \mathrm{g} / \mathrm{ml}$ in PBS $)$ for $10 \mathrm{~min}$ at room temperature.

After washing in buffer A, coverslips were mounted in 1:10 (v/v) mixture of PBS/glycerol containing $p$-phenylendiamine as antifading agent. Observation was performed by epifluorescence with a Nikon Eclipse 600 microscope equipped with a 100-W mercury lamp. The following conditions were used: 450-490 nm excitation filter (excf), $510 \mathrm{~nm}$ dichroic mirror (dm) and $520 \mathrm{~nm}$ barrier filter (bf) for FITC; 330-380 nm excf, $400 \mathrm{~nm} \mathrm{dm}$ and $420 \mathrm{~nm}$ bf for Hoechst 33342.

DNA static cytometry. Samples of NIHb and NIHs cells, grown on coverslips, after tubulin and DNA labelling, were used for static cytometry measurements. The amount of DNA per cell was evaluated by measuring the fluorescence emitted by each single nucleus of cells showing different microtubular alterations (e.g. paracrystals and bundles). For this analysis, a cytoflurometer Nikon P II (Nikon, Kanagawa, Japan) was used. In the cytometric apparatus, an appropriate system of diaphragms permitted the selection of single nuclei and the fluorescence was quantitatively analyzed after automatic subtraction of the background brightness.

For each experimental condition, three slides were examined and 300 cells per slide were measured. The results were represented as histograms of DNA content.

Transmission electron microscopy. NIHb and NIHs cells, grown in $75 \mathrm{~cm}^{2}$ culture flasks, in the various experimental conditions, were collected by centrifugation. Pellets were fixed with $2.5 \%$ glutaraldheyde in cacodylate buffer $(0.1 \mathrm{M}, \mathrm{pH} 7.4)$ for $2 \mathrm{~h}$. After washing in cacodylate buffer containing $6 \%$ 

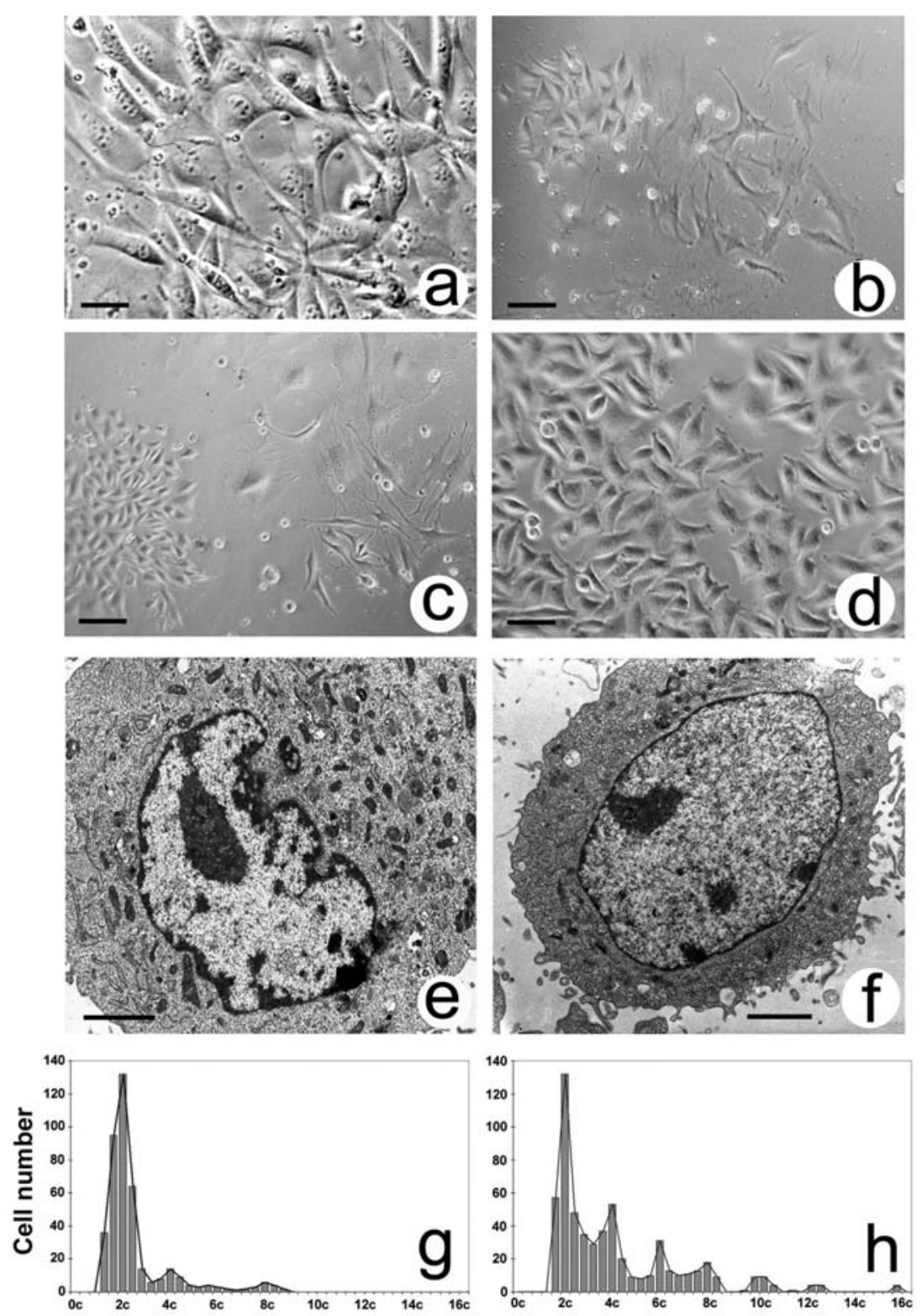

DNA content

Figure 1. Phase contrast microscopy of NIH/3T3 cells. (a) Morphological aspects of conventional culture (NIHb); (d) morphology changes of the culture after stress condition (NIHs). (b and c) Microscopic fields relative to NIH/3T3 cells re-fed with complete medium, after serum deprivation for 7 (b) and 10 days (c): areas showing cells with different morphology, typical of NIHs (left side of the images) or similar to NIHb cells (right side of the images). (e and f) Ultrastructure of $\mathrm{NIHb}(\mathrm{e})$ and NIHs cells (f). ( $\mathrm{g}$ and h) DNA content histograms of NIH/3T3 cells in basal condition (NIHb) and after selection of a stresssurviving subpopulation (NIHs). Bars: (a and d) $12 \mu \mathrm{m}$; (b and c) $24 \mu \mathrm{m}$; (e and f) $2 \mu \mathrm{m}$.

sucrose, the pellets were post-fixed in $1 \% \mathrm{OsO}_{4}$ in cacodylate buffer for $90 \mathrm{~min}$ at $4^{\circ} \mathrm{C}$. The cells were then resuspended in $1 \mathrm{ml} 2 \%$ agarose at $35^{\circ} \mathrm{C}$ and centrifuged at $300 \mathrm{~g}$ for $10 \mathrm{~min}$ at $35^{\circ} \mathrm{C}$. After $1 \mathrm{~h}$ at $4^{\circ} \mathrm{C}$, the samples were fragmented, dehydrated in an ethanol series and embedded in epoxy resin (Agar 100; Agar Scientific, Stansted, UK) at $60^{\circ} \mathrm{C}$ for $48 \mathrm{~h}$. Ultrathin sections were double stained with uranyl acetate and lead citrate, examined and photographed using a Zeiss EM 900 transmission electron microscope (Carl Zeiss Jena $\mathrm{GmbH}$, Jena, Germany), operating at $80 \mathrm{kV}$.

Reagents. Chemicals and antibodies, except where specified, were purchased from Sigma.

\section{Results}

Morphological and cytokinetic aspects of NIHb and NIHs cells in control conditions. Phase contrast microscopy analysis of NIH/3T3 cells permitted the demonstration of serum deprivation induced in the conventional culture $(\mathrm{NIHb})$, the selection and the persistence of cells with a reduced cytoplasm (NIHs) with respect to the cells constituting the original culture (Fig. 1). In the microscopic fields of Fig. 1b and c, areas with cells showing different morphology, relative to 7 (b) and 10 (c) days serum-deprived cultures and successively restored with complete medium, are shown. On the left side of Fig. $1 b$ and c, cells with reduced dimensions and with a morphological 
aspect typical of NIHs cells are visible. On the right side of Fig. $1 \mathrm{~b}$ and c, cells, usually present in the conventional culture $(\mathrm{NIHb})$, are also present. These latter cells progressively tend to detach from the growth substrate and to be lost.

The comparison between electron micrographs of $\mathrm{NIHb}$ (Fig. 1e) and NIHs (Fig. 1f) cells showed a different cytoplasm extension and complexity: in NIHs cells, a lower number of organelles and endomembranes was evident.

From a cytokinetic point of view, analysis of histograms relative to the DNA content cytofluorometry (Fig. $1 \mathrm{~g}$ and $\mathrm{h}$ ) demonstrated that the changes of cell morphology and ultrastructure were accompanied by an increase of the kinetic activity, in terms of cell proliferation, and the appearance of cells with higher ploidy levels (10c-16c) that did not show proliferative activity (Fig. 1).

\section{Vinblastine (VBL) treatment}

Fluorescence microscopy. In NIHb and NIHs cells, fluorescence microscopy after tubulin immunostaining permitted to show, in comparison with control conditions (Fig. 2a and d), the drastic changes induced by VBL (Fig. 2b, c, e and $f$ ).

In particular, in NIHb cells, incubation with the antimicrotubular agent induced in all cells the disappearance of the typical microtubular network. A small number of the cells showed depolymerization exclusively, while this alteration was accompanied, with different frequency, by the appearance of particular types of tubulin aggregations, such as paracrystals and spiral-like structures (Fig. 3). Morphological aspects of cells with paracrystals or spirals are shown in Fig. 2. Paracrystals are the consequence of a precise orientation of protofilaments arranged in parallel masses (see below ultrastructural analysis). VBL-induced spirals can be the result of a lateral aggregation of protofilaments, with helicoid arrangement. It must be underlined that the cells showed, in association with depolymerization, either paracrystals or spirals, exclusively. In NIHb cells, these cytoskeletal changes could be related to prominent nuclear anomalies such as $\mathrm{O}$ shaped (Fig. 2b) and lobulated nuclei. The appearance of micronucleations with high frequency was also observed (Fig. 3a).

In NIHs cells, VBL treatment caused the same type of microtubular alteration, even if helicoid tubulin aggregates (Fig. 2e and f) appeared with higher frequency with respect to paracrystals more expressed by NIHb cells (Fig. 3a). As far as NIHs nuclear aspects is concerned, the analysis performed by fluorescence microscopy after DNA fluorochromization showed, in comparison with NIHb cells, more apoptosis than micronucleations (Fig. 3a).

Ultrastructural analysis. Electron microscopy analysis in $\mathrm{NIHb}$ cells showed some ultrastructural features of alterations induced by VBL treatment at nuclear and cytoplasmic levels. Besides fragmentation/micronucleation phenomena (Fig. 4a), initial events of nuclear degeneration were shown: sometimes, there were budding and enlargement of the nuclear cisterna (Fig. 4b and c). These phenomena can be associated with the presence of tubulin paracrystals strictly near to the nucleus (Fig. 4b and c) with different orientation at the perinuclear area and dispersed in the cytoplasm, also near to
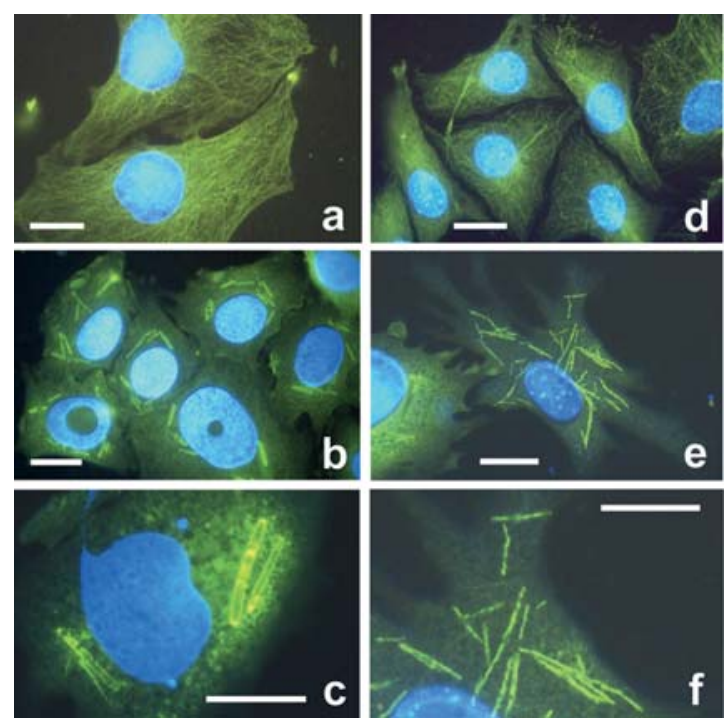

Figure 2. Double fluorescence (anti-tubulin/FITC and DNA-Hoechst 33342) in NIHb (a, b and c) and NIHs cells (d, e and f). (a and d) Controls; b, c, e and f) VBL treatment. In (b) and (c), tubulin paracrystals are visible, while in (e) and (f), spiral-like structures are shown. Bars: $6 \mu \mathrm{m}$.
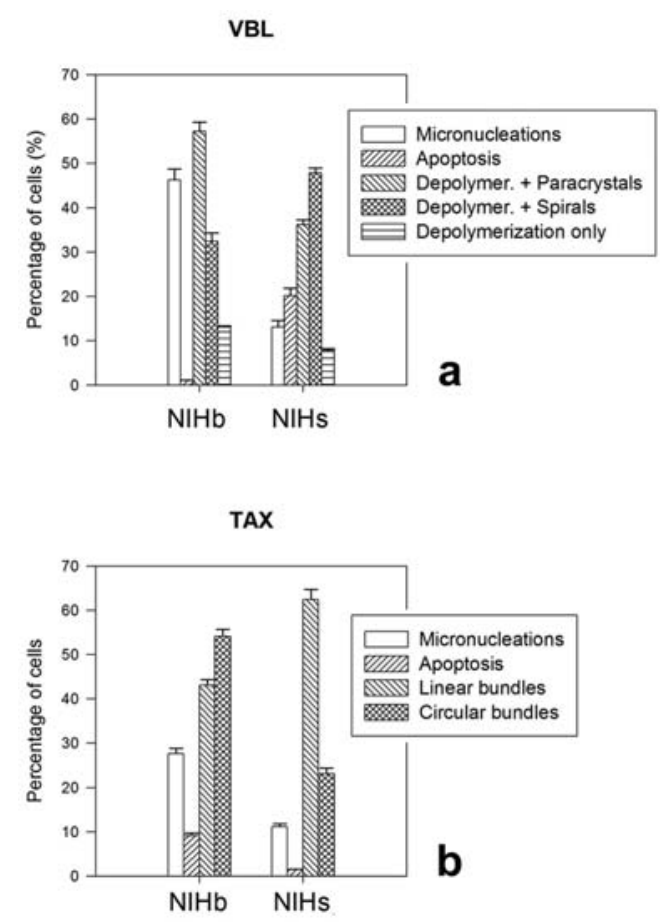

Figure 3. Percentage distribution of micronucleations, apoptosis and microtubular anomalies observed in VBL- (a) and TAX-treated (b) NIHb and NIHs cells. The data represent mean \pm SEM of triplicate experiments.

secondary lysosomes (Fig. 4a and d). Ultrastructural analysis permitted also to outline the simultaneous presence of tubulin paracrystals and massive storage of intermediate filament bundles, often distributed around the nucleus (Fig. 4d, e and f). At cytoplasm level, in some cases, there were residual bodies (Fig. 4a). In general, cisternae of endoplasmic reticulum were scarcely evident. 

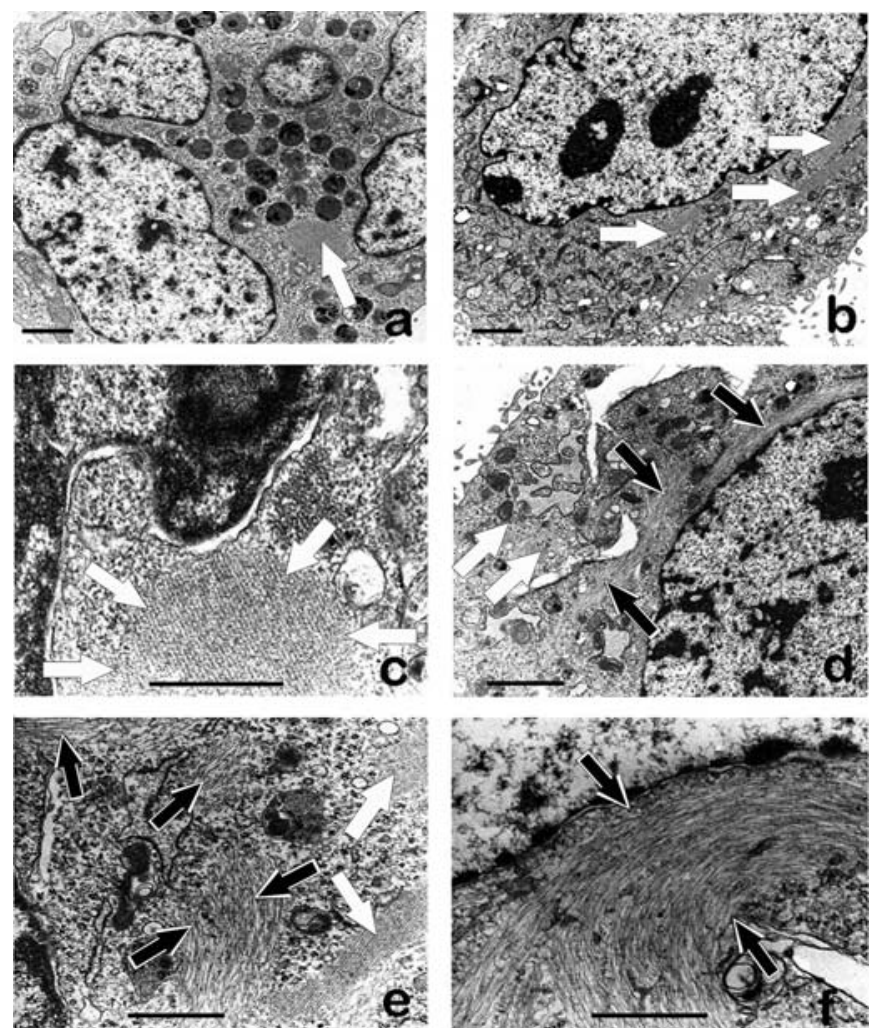

Figure 4. Ultrastructure of VBL-treated NIHb cells. Nuclear fragmentation/ micronucleation (a) and budding with enlargement of the nuclear cisterna (b and c) are shown. Tubulin aggregates (paracrystals: white arrows) and bundles of intermediate filaments (black arrows) surrounding the nucleus are also visible. Bars: a, b and $\mathrm{d} 2 \mu \mathrm{m}$; c, e and $\mathrm{f} 1 \mu \mathrm{m}$.

In NIHs cells, VBL treatment induced, at nuclear level, changes in part similar to those pointed out in NIHb cells, with higher dynamic phenomena of the nuclear envelope (Fig. 5a and b). At cytoplasm level, the appearance of enlarged cisternae of endoplasmic reticulum (Fig. 5b) and vesicles of possible autophagocytic origin (Fig. 5c) was observed; sometimes, these signs of cell suffering were associated with the presence of different forms of tubulin aggregation (Fig. 5b). In general, with respect to the basal condition (NIHb cells), a lower number of paracrystals was observed.

DNA static cytometry. Quantitative analysis of the DNA content, performed by static cytofluorometry, in cells showing paracrystals or spirals, permits to correlate, in NIHb and NIHs cultures, the appearance of these reaggregation forms of tubulin with the various phases of the cell cycle, also in relation to cell death (Fig. 6).

In NIHb cells, the comparison between the frequency histograms of the DNA fluorescence emission in the two subpopulations of cells with paracrystals and spirals demonstrated a similar outline (Fig. 6b). The analysis of DNA profiles showed not only peaks relative to the $\mathrm{G} 1$ and $\mathrm{G} 2 / \mathrm{M}$ (2c and 4c DNA content, respectively) cell cycle phases, but also a sharp intermediate peak indicative of degenerating or c-mitotic cells that can derive from the G2/M phase, as a consequence of the block of the spindle kinesis during the mitosis (Fig. 6b). The other two peaks, coinciding with $6 \mathrm{c}$ and 8c DNA levels, indicate the presence of polyploid cells in
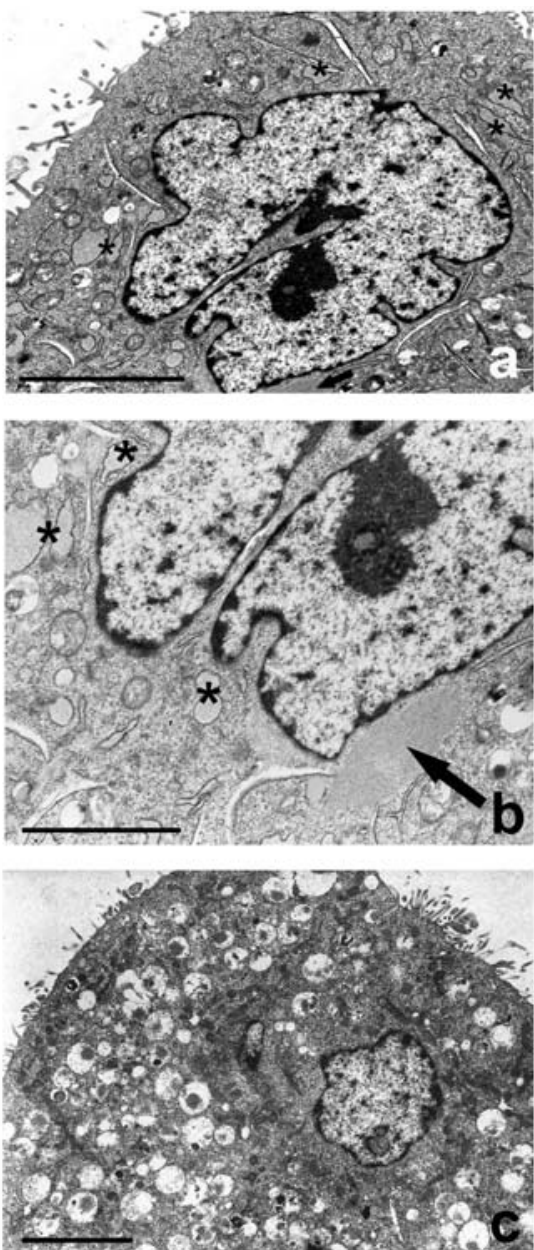

Figure 5. Ultrastructure of VBL-treated NIHs cells. Effects of microtubule destabilizing agent at cytoplasmic level: ( $a$ and $b$ ) presence of enlarged cisternae of endoplasmic reticulum (asterisks) and paracrystals (arrow); (c) large areas of vacuolization. Nuclear and cytoplasmic alterations appear to range from apoptosis to autophagic cell death. Bars: (a and c) $0.5 \mu \mathrm{m}$; (b) $0.25 \mu \mathrm{m}$.

addition to tetraploid elements, also containing paracrystals or spirals (Fig. 6b).

On the contrary, in VBL-treated NIHs cells, cytofluorometric profiles relative to the DNA fluorescence showed some differences in the two cell subpopulations containing paracrystals or spirals (Fig. 6e). The amount of cells in the G2/M phase showing paracrystals or macrotubules was comparable; on the contrary, the G1 cells showed histograms with different patterns. Apoptotic cells containing paracrystals appeared to originate from $2 \mathrm{c}$ and $4 \mathrm{c}$ cells, while apoptotic cells containing spirals showed a diploid origin.

\section{Taxol (TAX) treatment}

Fluorescence microscopy. In NIHb cells, treatment with the microtubule-stabilizing agent causes the formation of a notable number (Fig. 3b) of microtubular bundles with linear or circular arrangement around the nuclear area, often containing karyological fragments and micronucleations (Fig. 7a, b and c). In some cells, these changes coexist with the presence of several microtubule organizing centers (MTOCs). This latter event is particularly evident in mitotic cells and could play a role in cell death phenomena (mitotic catastrophe) (Fig. 8a 
NIHb
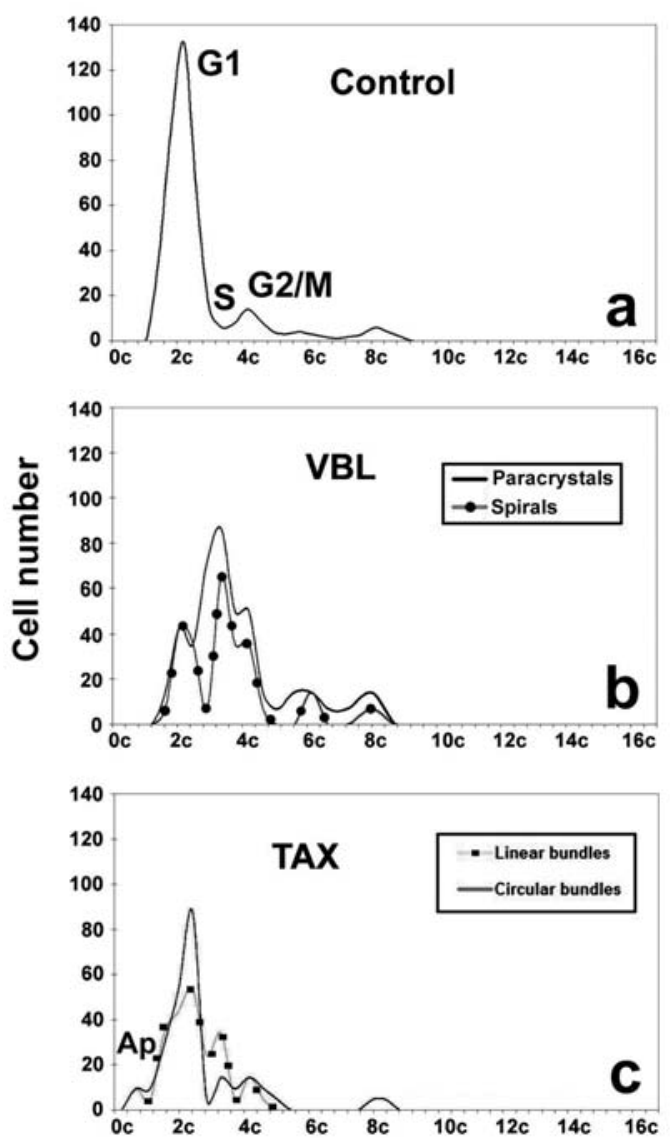

NIHs
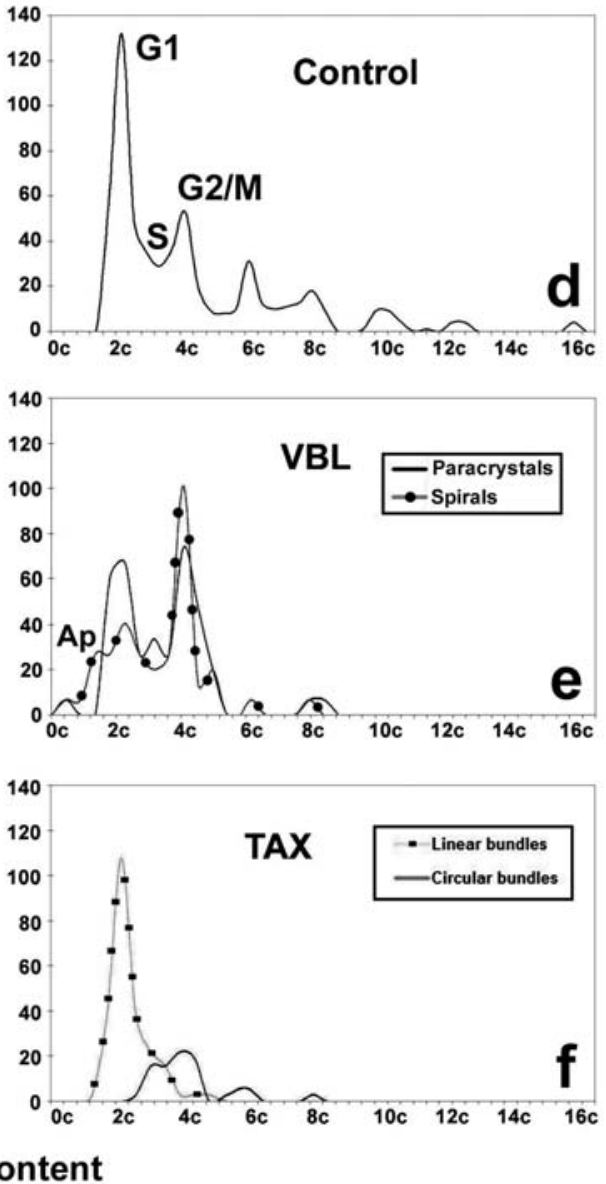

Figure 6. Typical static cytometric analysis of the DNA cellular content of NIHb (a, b and c) and NIHs cells (d, e and f) in control conditions (a and d) and after treatment with VBL (b and e) and TAX (c and f). In cultures incubated with the antimicrotubular agents, the DNA content has been evaluated in cells showing paracrystals or spiral-like structures (VBL treatment) and linear or circular bundles (TAX treatment). The curves represent the interpolation (see also Fig. $1 \mathrm{~g}$ and $\mathrm{h}$ ) of the values of frequency histograms. Beyond the cell cycle phases (G1, S and G2/M), only sub-2c apoptotic cells (Ap) are indicated.

and a'). Immunofluorescence analysis performed on NIHs cultures demonstrated that, in these cells, TAX induced less thick microtubule bundles (Fig. 7d, e and f) than those found in NIHb cells (Fig. 7a, b and c). In NIHs cells, the general distribution of the microtubular network appears to be in part maintained, even though it showed discontinuity in some cytoplasmic areas and at the cell periphery (Fig. 7e). Furthermore, these minor effects on microtubules are correlated with a reduced number of cells that degenerate by alternative cell death, subsequent micronucleation and/or die by apoptosis (Fig. 3b).

Ultrastructural analysis. In NIHb cells, TEM analysis demonstrates the presence of micronuclei (probably originated from mitotic alterations) in which chromatin hypercondensation can appear (Fig. 8b). At cytoplasmic level, the appearance of multiple centers from which microtubules originate is confirmed (Fig. 8c) and the presence of multivesicular bodies, probably representative of advanced stages of autophagic phenomena, is also visible (Fig. 8c).

In NIHs cells, nuclei appeared to be scarcely heterochromatic, while nucleoli showed high-condensation (Fig. 9a) and, in some cases, an altered morphology; nuclear fragmentation phenomena were scarce. At cytoplasm level, there were strong signs of degeneration, with the presence of vacuolization and vesicles containing portions of organelles or myelin figures (Fig. 9b). The presence of autophagosomes is indicative of active autolytic phenomena.

DNA static cytometry. Cytofluorometric analysis of the DNA content, related to cells with linear or circular microtubule bundles induced by TAX treatment, shows an opposite situation, by comparing NIHb vs. NIHs cultures (Fig. 6). In general, circular bundles are present with higher frequency in $\mathrm{NIHb}$ cells, in particular in the G1 phase of the cell cycle (Fig. 6c), while; in NIHs cells, they appear, with lower frequency, prevailingly in the S-G2/M phases and in polyploid cells (Fig. 6f). In NIHb cultures, the curve relative to DNA fluorescence of cells with linear microtubular bundles (Fig. 6c) shows a plurimodal pattern with peaks in correspondence of $\mathrm{G} 1, \mathrm{~S}$ and $\mathrm{G} 2 / \mathrm{M}$ phases. The peak relative to intermediate DNA content $(2 c-4 c)$ is probably enriched with hypo-4c micronucleated cells derived from the G2/M phase of the cell cycle. The appearance of microtubule bundles with linear arrangement appears to involve, in NIHs cells, in particular the G1 phase of the cell cycle (Fig. 6f). In these cells, the 

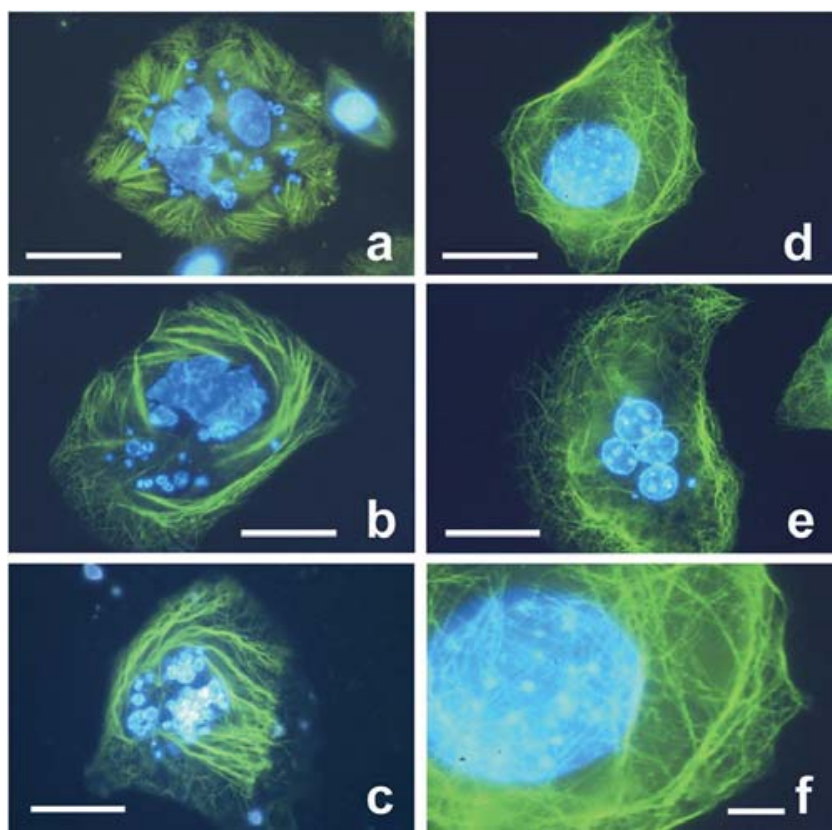

Figure 7. Double fluorescence (anti-tubulin/FITC and DNA-Hoechst 33342) in NIHb (a, b and c) and NIHs (d, e and f) cells after TAX treatment. In (a), short linear bundles of microtubules are visible, while in (b and $c)$, circular bundles of microtubules are shown. In (d) and (f), thin circular bundles are represented, in (e) discontinuity of the microtubular network is evident. Bars: (a-e) $6 \mu \mathrm{m}$; (f) $1.5 \mu \mathrm{m}$.
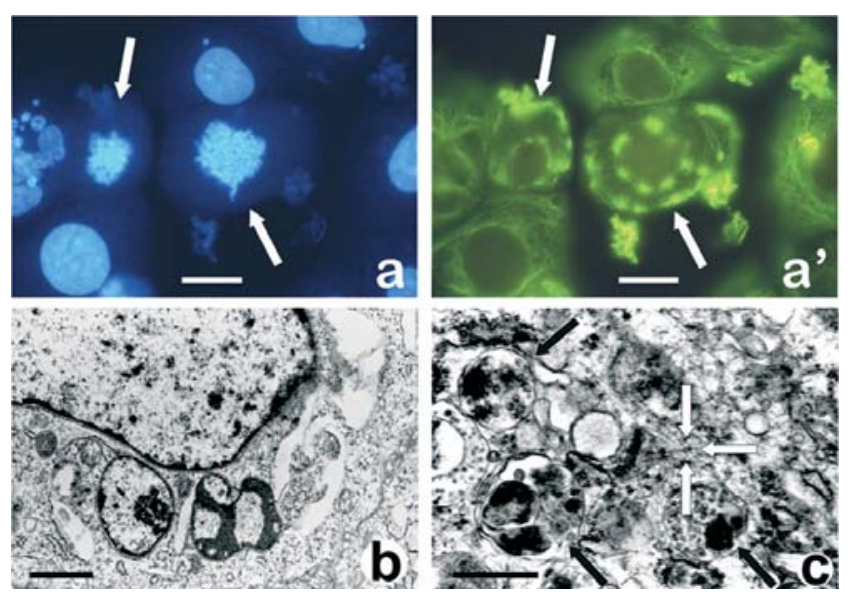

Figure 8. (a and a') Fluorescence microscopy of double-stained NIHb cells (a): DNA/Hoechst 33342; (a'): anti-tubulin/FITC) observed in two conditions of the specimen excitation. A correlation between aberrant mitoses and multiple MTOCs (arrows) could be established. (b and c) Ultrastructure of TAX-treated NIHb cells: (b) presence of micronuclei with chromatin hypercondensation; (c) cytoplasmic area in which an MTOC (white arrows) surrounded by multivesicular bodies (black arrows) is visible. Bars: (a and a') $6 \mu \mathrm{m}$; (b) $2 \mu \mathrm{m}$; (c) $0.5 \mu \mathrm{m}$.

microtubular changes could be related to a lower formation of micronuclei (Fig. 3b). As far as apoptosis derived from the G1 phase (hypo-2c areas) is concerned, the curves relative to DNA fluorescence show scarce signals, in cells with linear or circular bundles, in NIHb cultures only. It must be added that $\mathrm{NIHb}$ and NIHs cultures after treatment with the two drugs were characterized by the disappearance of cells with high ploidy levels (from 10c to 16c DNA content).
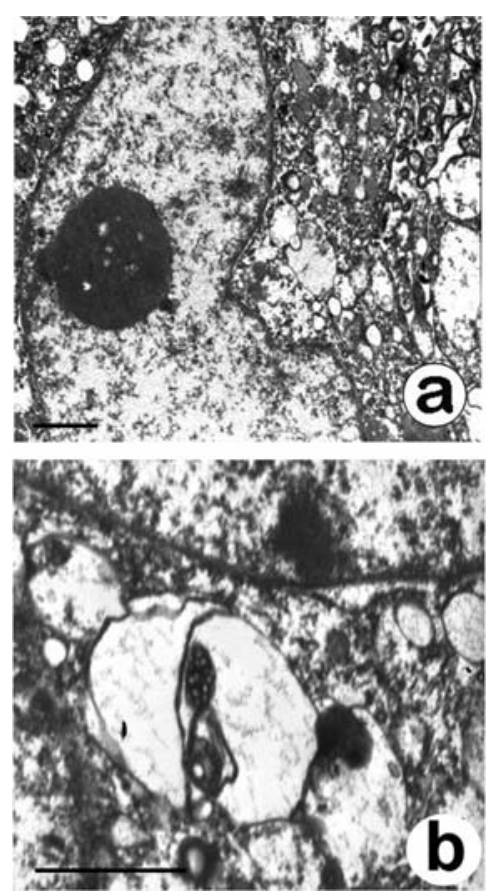

Figure 9. Ultrastructure of TAX-treated NIHs cells. Micrographs show the effects of the stabilizing microtubule agent especially at cytoplasmic level, with the appearance of wide areas of vacuolization (a) and some details of autophagocytic vacuoles (b). Bars: (a) $2 \mu \mathrm{m}$ and (b) $1 \mu \mathrm{m}$.

\section{Discussion}

In tumor treatment, chemotherapy is aimed to induce the elimination of neoplastic cells; unfortunately, cell cycle effects and death phenomena can involve, in different tissues, not only neoplastic cells, but also normal actively proliferating cells. The possibility of inducing selective death in neoplastic cells is related not only to the action mechanism(s) and pharmacological drug concentration, but also depend on the equilibrium of various biological factors, such as features of neoplastic cells, related to tumor cell heterogeneity. Fibroblastic cells and cell lines derived from solid tumors, such as sarcomas, characterized by well-organized cytoskeleton, can represent a pharmacological target model for the study of antimicrotubular drug action $(19,20)$. These drugs can induce the depolymerization or hyperpolymerization of microtubules, most likely by modifying their dynamics $(7,21)$, and consequently can block the cell cycle mostly during mitosis or produce abnormal mitoses. Metaphase spindle microtubules are strongly altered by inducing control factors that cause mitotic block; cells that are able to enter the G1 phase, despite damages, can repair, becoming polyploid, or die $(22,23)$.

We studied a mouse fibroblast NIH/3T3 line by comparing the action of antimicrotubular drugs in normal/ conventional (NIHb) and after critical culture conditions (NIHs). NIHs cells were obtained from NIHb cell line after a prolonged period of serum deprivation. This actively growing cell subpopulation, though maintaining a fibroblastoid morphology, showed lower size in the modal population, even though cells with high ploidy levels (up to 16c) were present in subconfluent cultures (18). The increase of polyploidy may represent an adaptative response to the stressful conditions of the culture microenvironment or a sign 
of genetic instability linked to a situation of aged cell-related cancer (24), in which change in ploidy can occur through endoreplication mechanism (25). In this cell model, we have compared the dynamics of microtubular network changes, induced by the treatment with microtubule destabilizing (Vinblastine, VBL) or stabilizing (Taxol, TAX) agents. The effects on cytoskeleton, related to micronucleation and death processes, have been quantified by evaluating their specific incidence during the various cell cycle phases.

$\mathrm{NIHb}$ and NIHs cells showed different responses to the antimicrotubular agents especially in the amount of anomalies induced at cytoskeletal and nuclear levels. As far as VBL is concerned, this drug caused, in NIHb and NIHs cells, microtubule depolymerization (in all the cells) and, with different frequency, two main anomalies of tubulin reaggregation: paracrystals and spiral-like structures. In particular, VBL caused the prevalence of paracrystals in $\mathrm{NIHb}$ cells, while in NIHs cells there was the predominance of spirals.

In several experimental studies, the effect of VBL on various cell types, such as cells of the kidney cortex (26) has been analysed. After VBL treatment, these cells showed a typical reduction of the number of cytoplasmic microtubules or their apparent absence. The formation of particular intracytoplasmic paracrystals, formed after binding of VBL to microtubular subunits that, instead of originating normal microtubules, formed intracytoplasmic tubulin crystalline aggregates (26-28) has been also observed. Despite numerous investigations indicating that Vinca alkaloids appear to have similar actions at the tubulin level (29), it must be underlined that different responses based on the relationship between cell type features and drug concentration can exist. In vitro, they all prevent microtubule assembly in the micromolar range (29). At higher concentrations, these compounds induce the assembly of tubulin spiral filaments in vivo and in vitro $(30,31)$ interacting laterally to form large ordered aggregates. Such aggregates are called paracrystals when their size and spiral order lead to birefringence properties under polarized light microscopy. Studies have described variable effects of temperature and MAPs on aggregate morphology induced by Vinca alkaloids (28). Other authors have suggested that the vincristine-induced spiral structures are related to the Vinca-induced paracrystal, the in vitro crystal formation observed in thin-sectioned samples corresponds to the aggregation of spirals seen in negatively stained material which occurs over a period of time after drug addition. In addition, it has been shown that MAPs are required for formation of crystals as well as spirals, further indicating that these structures are closely related (32).

In our experimental model, VBL treatment caused the appearance of the two aggregation forms of tubulin in $\mathrm{NIHb}$ and NIHs cells; nevertheless, it has been already underlined that paracrystals or spirals showed a different prevalence in the two cultures. This partial different response of NIHb and NIHs cells at cytoskeletal level was also correlated with different effects at nuclear level: in NIHb cells, there were prevalent micronucleation, as expression mainly of mitotic block (cmitosis), while in NIHs cultures, cell death with apoptotic/ autophagocytotic features appeared to be predominant. This can be interpreted by considering the cytofluorometric profiles of the DNA content in NIHs cells, after VBL treatment, that show, in comparison with NIHb cells, the presence of sub-2c and sub-4c values, more indicative of apoptosis in cells containing paracrystals. Furthermore, the fact that the G2/M block caused, in NIHs cells, a lower micronucleation could be explained by considering that microtubular network in these cells can have a different sensitivity in the various phases of the cell cycle favouring a different fate in relation to cell death. In particular, in NIHb cells, the interphasic microtubules and the mitotic ones could be differently sensitive also in relation to a marked interaction with the growth substratum and anchorage dependence. The higher incidence of micronucleations in NIHb cells could be also related to the phenomena of dynamism of the nuclear envelope, shown by electron microscopy. In other studies, it has been already demonstrated that micronuclei can originate as a consequence of not only mitosis alterations (c-mitosis), but also budding of the nuclear cisterna $(18,33)$. In VBL-treated $\mathrm{NIHb}$ cells, ultrastructural data show the presence of tubulin paracrystals strictly near to the nuclear cisterna; in the dynamics of these events, the reorganization of intermediate filaments (vimentin) could also play a role; they appear to be distributed in compact bundles around the nucleus and perhaps interacting with tubulin paracrystal structures.

By comparing VBL effects on NIHb and NIHs cultures, it appears to emerge that cells with the same origin, that acquire different phenotypic features (NIHs vs. NIHb cells), can show a partial different response to the same drug, a situation like that occurring during the neoplastic transformation. Nevertheless, the growth condition that favours the kind of tubulin reaggregation and consequently the possible different way of cell degeneration must be defined. In NIHb and NIHs cells, beside depolymerization, we observed the presence of paracrystals and spiral-like structures; nevertheless, we emphasize that individual cells show either the one or the other form of tubulin reaggregation. This fact could indicate the existence of specific subcellular conditions that are able to favour the formation of paracrystals or spirals. In this field, beyond specific MAPs expression, other factors and cytoplasmic conditions could play an important role. Some authors have demonstrated that by reducing intracellular $\mathrm{pH}$ in proximity to neutrality, there was a significant acceleration of VBL-induced paracrystal growth. When the cytoplasmic $\mathrm{pH}$ value ranged from 6.3 to 6.7 , paracrystals disappeared and short and thick tubulin bundles appeared (34). The variability of these cytoplasmic factors could be correlated, in the same culture, with the metabolic activity, in a specific phase of the cell cycle and with the cell kinetics in terms of proliferation and polyploidization.

The results obtained in NIHb cultures, after TAX treatment, confirm that these cells are more susceptible to induction of micronuclei, although the effects on the microtubular network are different from those induced by VBL. This drug caused in NIHb cells the appearance of circular bundles that showed a typical arrangement around the nucleus or micronuclei/nuclear fragments. In these cells, micronucleation events are also probably favoured by the presence of multipolar mitoses induced by the drug. A contribution to the presence of nuclear fragments in degenerating cells could also derive by the particular 
disposition of circular microtubule bundles. The DNA cytofluorometric analysis indicates, especially in NIHs cells, that this microtubular anomaly appears to involve the cells with a DNA content higher than 2c (tetraploid or polyploid cells). These data underline how the efficacy of the pharmacological treatment must take in account the cell cycle phase in which the cells are and the polyploidy conditions. Moreover, in NIHs cells, the appearance of microtubule bundles with linear arrangement appears to be involved in particular the G1 phase of the cell cycle; these microtubular changes could be related not only to a lower formation of micronuclei, but also to scarce cell dynamism, that could negatively influence conventional apoptotic processes derived from this phase of the cell cycle. A potential role for microtubules, in cooperation with actinmyosin II, has been described in the formation of chromatinrich surface blebs of the late apoptotic cells. The apparent close association between microtubules and condensed chromatin in late apoptotic cells prompted some authors to consider that microtubules contribute to apoptotic chromatin remodelling and, in general, cell dynamism during apoptosis. Data from literature suggest that the interphase microtubule network is disassembled early in the execution phase and that, successively, it is replaced by an apoptotic microtubule array at later stages (35).

In our experimental model, as far as apoptosis derived from the G1 phase (hypo-2c areas) is concerned, the curves relative to DNA fluorescence show scarce signals, in cells with linear or circular bundles, in NIHb cultures only. By comparing TAX vs. VBL treatment in NIHs cells, the data obtained showed a decrease of apoptosis induced by the stabilizing agent. This could demonstrate a particular resistance of NIHs cells to TAX-derived drugs and could be in agreement with other studies that reported the isolation of cultured mammalian cells resistant to paclitaxel (36). Although the relevance of tubulin mutations to clinical resistance has been called into question (37), mutant cell lines have proven useful in making predictions about amino acid residues that may be involved in drug binding (38-40) and in mediating potential interactions between tubulin subunits or between microtubules and associated molecules $(41,42)$. Other studies previously proposed a model to explain the drug resistance mechanism in these cells based on the concept that microtubules function normally within a narrow range of microtubule assembly or stability (43). Tubulin mutations that increase microtubule assembly or stability produce resistance to drugs that inhibit assembly; whereas mutations that decrease tubulin assembly or stability produce resistance to drugs that enhance assembly. Consistent with this model, all paclitaxel-resistant cell lines have diminished microtubule assembly, while all colcemid-resistant cell lines have increased microtubule assembly (44). The exact mechanism by which mutations in tubulin genes affect microtubule assembly is not known. Alterations occur in various regions of $\alpha$ - or $\beta$-tubulin subunits, but again could cause structural changes that perturb subunit interactions to strengthen (colcemid-resistance mutations) or weaken (paclitaxel-resistance mutations) the microtubule network. Consistent with this interpretation, in some studies, immunofluorescence microscopy of paclitaxeldependent cells reveals a sparse microtubule network and defective mitotic spindles (45). Moreover, a study has demonstrated significantly increased dynamic instability in a paclitaxel-dependent cell line (46).

In our cell model, in some cells of NIHs cultures, Taxol did not induce the appearance of microtubule bundles, but the partial maintenance of the microtubular network. This variability could be correlated with a different reactivity of the cells during the phases of the cell cycle. From this point of view, the relationship between microtubule assembly and cell proliferation and death must be defined. Drug-resistant cells have small changes in tubulin assembly that produce little or no effect on cell growth and survival, whereas drug-dependent cells have larger changes in assembly that produce clear defects in chromosome segregation and cell division and ultimately cause cell death (47). Poised between drug resistance and dependence are cells with intermediate alterations in microtubule assembly that produce partial drug dependence and a significant decrease in cell survival. These latter cells, along with paclitaxel-dependent cells rescued with a minimal concentration of the drug, help to define the limits of how far microtubule assembly can be altered before effects on cell division are encountered. Analysis of these various cell lines suggests that microtubule function is maintained when tubulin assembly is reduced or elevated by up to $45-50 \%$, thereby defining a 'normal range' of microtubule assembly that is able to support cell proliferation. Beyond those limits, microtubule function is compromised and cells fail to survive. Therefore, the unequal alteration of the microtubular network and consequently of the cell kinetics and death, in our two culture cell lines, is probably due to different subtypes of microtubule-associated proteins and/or tubulin sub-types, derived from DNA-mutations and/or post-translational changes (48). The same hypothesis could be applied as regard the disappearing, after treatment, of the culture fraction with the higher ploidy levels (10c-16c).

In general, in our cell model, treatment with antimicrotubular agents, characterized by different mechanisms, after stress condition (NIHs cells), induced lower cell death phenomena than in the basal condition (NIHb cells); this fact was evident especially after TAX treatment, and was related to a discrete maintenance of the microtubular network and a lower number of microtubule bundles. This could be also related, beyond a probable different microtubule dynamism, to intracellular environment conditions in NIHs cells. These cells expressed some features observed in transformed cells, such as low number of mitochondria and a consequent more anaerobic metabolism. This was also testified by the release of acid catabolites, able to rapidly reduce the $\mathrm{pH}$ value of the culture medium. Our data demonstrated that drugs affecting the microtubular network could produce effects, related to programmed cell death, that could be expressed, in a manner quantitatively different, independence on the phases of the cell cycle and the general morphofunctional and metabolic features of the cells.

\section{References}

1. Rowinsky EK and Donehower RC: Systemic chemotherapy. In: The Chemotherapy Source Book. Perry MC (ed). 2nd edition, Williams and Wilkins, Baltimore, pp116-140, 1996.

2. Haskell CM: Antineoplastic agents: cancer treatment. In: Cancer Treatment. Haskell CM (ed). 4th edition, W.B. Saunders Co., Philadelphia, pp78-165, 1995. 
3. Schiff PB and Horwitz SB: Taxol stabilizes microtubules in mouse fibroblast cells. Proc Natl Acad Sci USA 77: 1561-1565, 1980.

4. Bollag DM, McQueney PA, Zhu J, Hensens O, Koupal L, Liesch J, Goetz M, Lazarides E and Woods CM: Epothilones, a new class of microtubulestabilizing agents with a Taxol-like mechanism of action. Cancer Res 55: 2325-2333, 1995.

5. ter Haar E, Kowalski RJ, Hamel E, Lin CM, Longley RE, Gunasekera SP, Rosenkranz HS and Day BW: Discodermolide, a cytotoxic marine agent that stabilizes microtubules more potently than Taxol. Biochemistry 35: 243-250, 1996.

6. Jordan MA, Toso RJ, Thrower D and Wilson L: Mechanism of mitotic block and inhibition of cell proliferation by Taxol at low concentrations. Proc Natl Acad Sci USA 90: 9552-9556, 1993.

7. Jordan MA, Thrower D and Wilson L: Effects of vinblastine, podophyllotoxin and nocodazole on mitotic spindles. Implications for the role of microtubule dynamics in mitosis. J Cell Sci 102: 401-416, 1992.

8. Fan M, Goodwin ME, Birrer MJ and Chambers TC: The c-Jun $\mathrm{NH} 2$-terminal protein kinase/AP-1 pathway is required for efficient apoptosis induced by vinblastine. Cancer Res 61: 4450-4458, 2001.

9. Moos PJ and Fitzpatrick FA: Taxanes propagate apoptosis via two cell populations with distinctive cytological and molecular traits. Cell Growth Differ 9: 687-697, 1998.

10. Woods CM, Zhu J, McQueney PA, Bollag D and Lazarides E: Taxol-induced mitotic block triggers rapid onset of a p53independent apoptotic pathway. Mol Med 1: 506-526, 1995.

11. Lieu CH, Chang YN and Lai YK: Dual cytotoxic mechanisms of submicromolar Taxol on human leukemia HL-60 cells. Biochem Pharmacol 53: 1587-1596, 1997.

12. Giannakakou P, Robey R, Fojo T and Blagosklonny MV: Low concentrations of paclitaxel induce cell type-dependent p53, p21 and G1/G2 arrest instead of mitotic arrest: molecular determinants of paclitaxel-induced cytotoxicity. Oncogene 20: 3806-3813, 2001.

13. Torres K and Horwitz SB: Mechanisms of Taxol-induced cell death are concentration dependent. Cancer Res 58: 3620-3626, 1998.

14. Fan W, Cheng LR, Norris JS and Willingham MC: Glucocorticoids selectively inhibit paclitaxel-induced apoptosis in a human breast cancer cell line. Cell Pharmacol 3: 435-440, 1996.

15. Fan W, Schandl CA, Cheng LR, Norris JS and Willingham MC: Glucocorticoids modulate Taxol cytotoxicity in human solid tumor cells. Cell Pharmacol 3: 343-348, 1996.

16. Jung M, Grunberg S, Timblin C, Buder-Hoffman S, Vacek P, Taatjes DJ and Mossman BT: Paclitaxel and vinorelbine cause synergistic increases in apoptosis but not in microtubular disruption in human lung adenocarcinoma cells (A-549). Histochem Cell Biol 121: 115-121, 2004.

17. Huang Y, Fang Y, Jinmin Wu J, Dziadyk JM, Zhu X, Sui M and Fan W: Regulation of Vinca alkaloid-induced apoptosis by NF$\mathrm{KB} / \mathrm{IKB}$ pathway in human tumor cells. Mol Cancer Ther 3: 271-277, 2004.

18. Spano A, Monaco G, Barni S and Sciola L: Cisplatin treatment of NIH/3T3 cultures induces a form of autophagic death in polyploid cells. Histol Histopathol 23: 717-730, 2008.

19. LaPensee EW, Reddy SP, Hugo ER, Schwemberger SJ and Ben-Jonathan N: LS14 cells: a model for chemoresistance in liposarcoma. Cancer Biol Ther 6: 519-524, 2007.

20. Díaz-Montero CM and McIntyre BW: Acquisition of anoikis resistance in human osteosarcoma cells does not alter sensitivity to chemotherapeutic agents. BMC Cancer 5: 39-48, 2005.

21. Toso RJ, Jordan MA, Farrell KW, Matsumoto B and Wilson L: Kinetic stabilization of microtubule dynamic instability in vitro by vinblastine. Biochemistry 32: 1285-1293, 1993.

22. Jordan MA, Wendell K, Gardiner S, Derry WB, Copp H and Wilson L: Mitotic block induced in HeLa cells by low concentrations of paclitaxel (Taxol) results in abnormal mitotic exit and apoptotic cell death. Cancer Res 56: 816-825, 1996.

23. Nitta M, Tsuiki H, Arima Y, Harada K, Nishizaki T, Sasaki K, Mimori T, Ushio Y and Saya H: Hyperploidy induced by drugs that inhibit formation of microtubule promotes chromosome instability. Genes Cells 7: 151-162, 2002.

24. Hoz C and Baroja A: Proliferative behaviour of high-ploidy cells in two murine tumor lines. J Cell Sci 104: 31-36, 1993.

25. Walen KH: Origin of diplochromosomal polyploidy in nearsenescent fibroblast cultures: heterochromatin, telomeres and chromosomal instability(CIN). Cell Biol Int 31: 1447-1455, 2007.
26. Tyson GE and Bulger RE: Vinblastine-induced paracrystals and unusually large microtubules (macrotubules) in rat renal cells. Z Zellforsch 141: 443-458, 1973.

27. Bensch KG and Malawista SE: Microtubular crystals in mammalian cells. J Cell Biol 40: 95-107, 1969.

28. Verdier-Pinard $\mathrm{P}$, Garès $\mathrm{M}$ and Wright $\mathrm{M}$ : Differential in vitro association of Vinca alkaloid-induced tubulin spiral filaments into aggregated spirals. Biochem Pharmacol 58: 959-971, 1999.

29. Lobert S, Vulevic B and Correia JJ: Interaction of Vinca alkaloids with tubulin: A comparison of vinblastine, vincristine, and vinorelbine. Biochemistry 35: 6806-6814, 1996.

30. Himes RH: Interactions of the Catharanthus (vinca) alkaloids with tubulin and microtubules. Pharmacol Ther 51: 257-267, 1991.

31. Hamel E: Interaction of tubulin with small ligands. In: Microtubule Proteins. Avila J (ed). CRC Press, Boca Raton, FL, pp89-191, 1990

32. Haskin KM, Donoso JA and Himes RH: Spirals and paracrystals induced by Vinca alkaloids: evidence that microtubule-associated proteins act as polycations. J Cell Sci 47: 237-247, 1981.

33. Fenech M: Cytokinesis-block micronucleus assay evolves into a 'cytome' assay of chromosomal instability, mitotic dysfunction and cell death. Mut Res 600: 58-66, 2006.

34. Tafanari H, Morita J, Yamanaka H, Yada K, Takahashi A and Izutsu K: Effects of acidic $\mathrm{pH}$ on the formation of vinblastineinduced paracrystals in Chinese hamster ovary cells. Biol Cell 82: 51-57, 1994.

35. Moss DK, Betin VM, Malesinski SD and Lane JD: A novel role for microtubules in apoptotic chromatin dynamics and cellular fragmentation. J Cell Sci 119: 2362-2374, 2006.

36. Casazza AM and Fairchild CR: Paclitaxel (Taxol): mechanisms of resistance. Cancer Treat Res 87: 149-171, 1996.

37. Sale S, Sung R, Shen P, Yu K, Wang W, Duran GE, Kim J, Fojo T, Oefner PJ and Sikic BI: Conservation of the class I btubulin gene in human populations and lack of mutations in lung cancers and paclitaxel-resistant ovarian cancers. Mol Cancer Ther 1: 215-225, 2002 .

38. Giannakakou P, Sackett DL, Kang Y-K, Zhan Z, Buters JTM, Fojo T and Poruchynsky MS: Paclitaxel-resistant human ovarian cancer cells have mutant $\beta$-tubulins that exhibit impaired paclitaxel driven polymerization. J Biol Chem 272: $17118-17125,1997$

39. Giannakakou P, Gussio R, Nogales E, Downing KH, Zaharevitz D, Bollbuck B, Poy G, Sackett D, Nicolaou KC and Fojo T: A common pharmacophore for epothilone and taxanes: molecular basis for drug resistance conferred by tubulin mutations in human cancer cells. Proc Natl Acad Sci USA 97: 2904-2909, 2000.

40. Hua XH, Genini D, Gussio R, Tawatao R, Shih H, Kipps TJ, Carson DA and Leoni LM: Biochemical genetic analysis of indanocine resistance in human leukemia. Cancer Res 61: 7248-7254, 2001.

41. He L, Yang CH and Horwitz SB: Mutations in B-tubulin map to domains involved in regulation of microtubule stability in epothilone resistant cell lines. Mol Cancer Ther 1: 3-10, 2001.

42. Kavallaris M, Tait AS, Walsh BJ, He L, Horwitz SB, Norris MD and Haber M: Multiple microtubule alterations are associated with Vinca alkaloid resistance in human leukemia cells. Cancer Res 61: 5803-5809, 2001

43. Cabral F, Brady RC and Schibler MJ: A mechanism of cellular resistance to drugs that interfere with microtubule assembly. Ann NY Acad Sci 466: 745-756, 1986.

44. Minotti AM, Barlow SB and Cabral F: Resistance to antimitotic drugs in Chinese hamster ovary cells correlates with changes in the level of polymerized tubulin. J Biol Chem 266: 3987-3994, 1991

45. Cabral F, Wible L, Brenner S and Brinkley BR: Taxol requiring mutant of Chinese hamster ovary cells with impaired mitotic spindle assembly. J Cell Biol 97: 30-39, 1983.

46. Goncalves A, Braguer D, Kamath K, Martello L, Briand C, Horwitz S, Wilson L and Jordan MA: Resistance to Taxol in lung cancer cells associated with increased microtubule dynamics. Proc Natl Acad Sci USA 98: 11737-11741, 2001.

47. Cabral F and Barlow SB: Resistance to antimitotic agents as genetic probes of microtubule structure and function. Pharmacol Ther 52: 159-171, 1991.

48. Esteve MA, Carrè M, Bourgarel-Rey V, Kruczynski A, Raspaglio G, Ferlini C and Braguer D: Bcl-2 down-regulation and tubulin subtype composition are involved in resistence of ovarian cancer cells to vinflunine. Mol Cancer Ther 5: 2824-2833, 2006. 